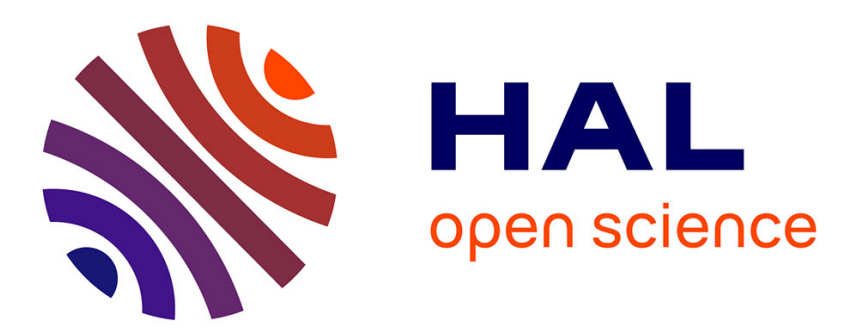

\title{
Combined analytical models for sound generation and transmission in cambered axial-flow outlet guide vanes
}

Michel Roger, Benjamin François

\section{To cite this version:}

Michel Roger, Benjamin François. Combined analytical models for sound generation and transmission in cambered axial-flow outlet guide vanes. European Journal of Mechanics - B/Fluids, 2017, 61, pp.218-225. 10.1016/j.euromechflu.2016.10.006 . hal-01724953

\section{HAL Id: hal-01724953 \\ https://hal.science/hal-01724953}

Submitted on 15 Nov 2018

HAL is a multi-disciplinary open access archive for the deposit and dissemination of scientific research documents, whether they are published or not. The documents may come from teaching and research institutions in France or abroad, or from public or private research centers.
L'archive ouverte pluridisciplinaire HAL, est destinée au dépôt et à la diffusion de documents scientifiques de niveau recherche, publiés ou non, émanant des établissements d'enseignement et de recherche français ou étrangers, des laboratoires publics ou privés. 


\title{
Combined analytical models for sound generation and transmission in cambered axial-flow outlet guide vanes
}

\author{
Michel Roger *, Benjamin François \\ Univ. Lyon, École Centrale de Lyon, Laboratoire de Mécanique des Fluides et Acoustique, UMR CNRS 5509,36 avenue Guy de Collongue, 69134 Ecully, France
}

\begin{abstract}
A new analytical approach is proposed to predict sound transmission through a row of outlet guide vanes taking into account the curvature and the inclination of the vanes. The approach couples two analytical tools, namely a mode-matching procedure iteratively applied at the leading-edge and trailing-edge interfaces, and an approximate transmission model inside the inter-vane channels. Both curvature and inclination are shown to have a significant effect on the reflection and transmission coefficients. They are addressed separately for the sake of a better understanding of the involved mechanisms. The complete chaining of the models is out of the scope of the paper. The interest of the approach is that it could be extended in a three-dimensional annular geometry to address sound generation and transmission problems.
\end{abstract}

\section{Introduction}

Aerodynamic noise from rotating blade architectures is a matter of concern in many areas of engineering sciences, such as aeronautical propulsion systems, ventilation and air conditioning units and so on. Facing more and more stringent regulations, its reduction is a crucial need for manufacturers, which also motivates the development of efficient prediction methods. In ducted axial-flow turbomachines the simplest configuration to deal with is made of a rotor and a stator, with often large numbers of blades and vanes. Two kinds of aeroacoustic mechanisms are involved, namely sound generation by the rotor and the stator because of their mutual interaction or the interaction with inflow distortions, and sound transmission through one element from sources located on the other element. This complexity makes the numerical solving of gas-dynamics equations for acoustic purposes a considerable task, well beyond the scope of affordable efforts in many practical cases. At the preliminary design stage or for the sake of a better physical understanding, addressing only a part of the physics with analytical approaches at the price of acceptable simplifications is a relevant alternative. Goldstein's formulation of the acoustic analogy derived by Ffowcs Williams \& Hawkings $[1,2]$ is generally used to model the sound-generation mechanisms

\footnotetext{
* Corresponding author.

E-mail address: michel.roger@ec-lyon.fr (M. Roger).
}

by a two-step procedure. The analogy states that the dominant sources of aerodynamic noise are the lift fluctuations induced on the blades and the vanes by the aerodynamic unsteadiness, acting as equivalent dipoles. The first step is therefore to determine the strength of the dipoles. The second step is to calculate their radiation by using the formalism of Green's functions. In contrast addressing sound transmission is only a matter of acoustic propagation in the presence of obstacles, which is equivalent to solve a diffraction problem in the presence of flow. In that context, the present work is aimed at deriving analytically the response of a row of outlet guide vanes (OGV) to incident acoustic waves, taking into account as many design features as possible. Considering only the response of the stator to acoustic excitation apparently reduces the scope of the study to the downstream transmission of rotor noise, caused for instance by the passage of the rotating blades through some inflow distortion or ingested turbulence. But the same formulation can be used to model the noise generated as boundary-layer turbulence is scattered at the trailing edges of the stator vanes, referred to as trailing-edge noise. Indeed the actual sources of that noise can be reproduced by acoustic dipoles approached very close to the trailing edges from downstream, as shown by Roger et al. [3]. By virtue of this equivalence trailing-edge noise radiation is interpreted as the upstream transmission of acoustic waves through the stator. Generalizing this idea makes the present formulation promising and versatile. It must be understood as a first step towards a unified approach for sound-generation and sound-transmission modeling in turbomachinery stages. 

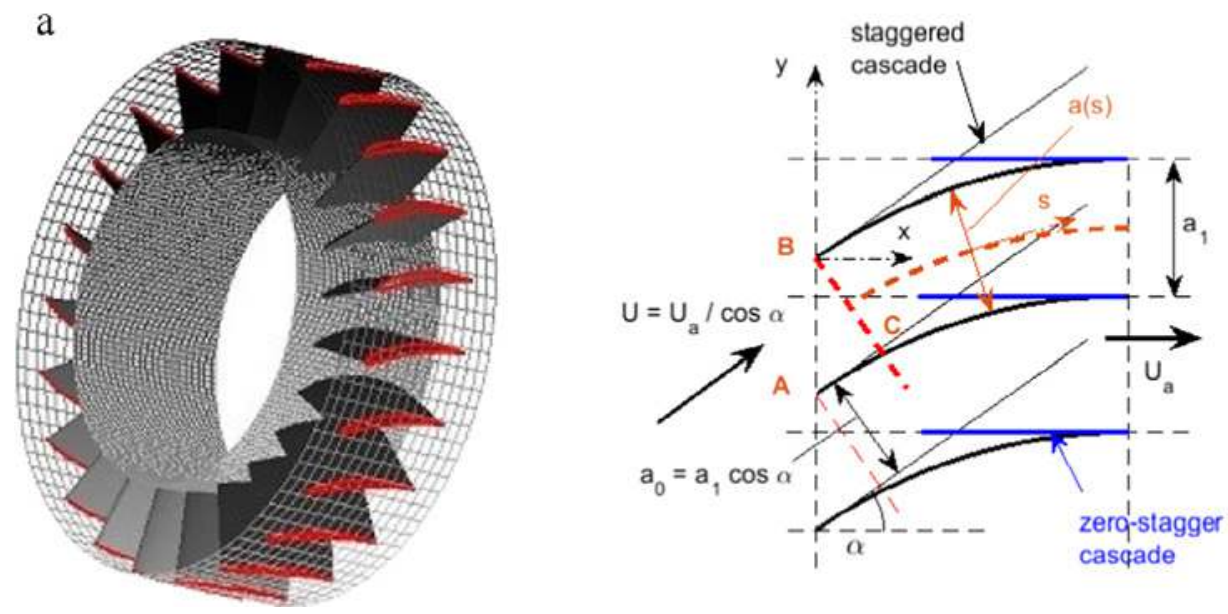

Fig. 1. (a) Typical configuration of axial-flow outlet guide vanes. (b) Unwrapped two-dimensional cascade representation introducing all notations used in the paper. Curvilinear abscissa along the dashed arc.

The addressed stator design is fully subsonic and depicted in Fig. 1(a). It is representative of the fan-OGV system of most turbofan engines in approach conditions or of subsonic fans used in aircraft air-conditioning systems. The vanes are cambered in such a way that they have a significant stagger at the leadingedge whereas they are axially oriented at the trailing edge, as emphasized by the two-dimensional unwrapped representation in Fig. 1(b). As a result the individual inter-vane channels are featuring bent ducts. This configuration allows recovering the swirl induced by the upstream rotor. The point is that analytical models used in the literature usually assimilate the vanes to flat plates of zero camber for mathematical tractability, which introduces arbitrariness in the choice of the equivalent flat-plate stagger angle and associated inaccuracy. For the assessment of wakeimpingement noise generation the induced lift forces are known to concentrate at the leading edges of the vanes. Therefore the latter are staggered according to the obliqueness of their mean camber line at leading-edge, accepting a nonrealistic representation of the trailing edge. A reasonably good agreement can be found between predicted and measured sound spectra upstream of the stator but substantial discrepancies are sometimes found downstream, as reported by Posson et al. [4] using a cascade response function derived by the Wiener-Hopf technique. If the dominant induced lift forces concentrate at the trailing edge, the vanes are better assumed aligned with the axis. This choice was made in a problem statement based on a mode-matching technique by Roger et al. [3] dealing with the trailing-edge noise of a cascade of outlet guide vanes (see also Bouley et al. [5]). But the sound transmission upstream of the stator is not reproduced accurately. To cope with this ambiguity, de Laborderie et al. [6] proposed a simple but quite empirical correction to Posson et al.'s model which confirms that the chosen flat-plate angle is a crucial parameter. This motivated the authors in re-addressing the problem of stator response in such a way that the aforementioned shortcomings are solved, in particular by accounting for different stagger angles at leading edge and at trailing edge on a more reliable basis. The theoretical background is now based on an iterative modematching procedure, considering the inter-vane channels as a periodic array of curved bifurcated waveguides. It can be outlined as follows. The open areas upstream and downstream of the stator, on the one hand, and the inter-vane channels, on the other hand, are considered as subdomains in which the local acoustic field is described by a sum of characteristic modes. The continuity of the complete field is imposed at the interfaces between subdomains in accordance with linearized conservations laws of gas dynamics. This provides systems of linear equations relating the modal coefficients. The transmission of the modes through the inter-vane channels is estimated with a model that accounts for variations of the modal parameters between the inlet and outlet crosssections of the channels. Based on the geometrical approximations introduced in Section 1, the procedure involves three models, two of which are addressed in the paper. As a first original contribution, the formulation of the mode-matching at the stator inlet is presented in Section 2. The mode-matching at the stator outlet for a zero stagger is not addressed here because it has been fully described in the Ref. [3]. But the case of staggered flat plates of finite length is also discussed in Section 2. The second contribution is the simplified sound-transmission model through the intervane channels of variable cross-section, detailed in Section 3. Sample results are given to highlight separately the effects of vane inclination and vane curvature but the chaining of the models is out of the scope of the paper. Its implementation is still in progress.

\section{Geometrical simplifications and general solving procedure}

The unwrapped representation of a cylindrical cut of the stator at some radius $r_{0}$ is shown in Fig. 1(b) as an infinite rectilinear cascade. Each vane is assumed of zero thickness and perfectly rigid; it is reduced to its mean camber line. The tangential vane-to-vane distance is $a_{1}=2 \pi r_{0} / V, V$ standing for the number of vanes. The stagger angles are $\alpha$ at the leading edge and zero at the trailing edge. In this ideal view the incident mean-flow direction has the angle $\alpha$ at the inlet and is parallel to the axis at the outlet. The inter-vane channels largely overlap. Their actual width is $a_{0}$ at inlet and $a_{1}$ at outlet with $a_{0}=a_{1} \cos \alpha$. At the leading edge and down to point $C$ in Fig. 1(b) the mean-camber lines of the vanes can be assimilated to the tangent staggered semi-infinite plates, the small error being negligible with regard to the ignored thickness of the vanes (see Section 3 for details). The local continuity of the sound field at this interface ignoring the complementary one can be described using the specific mode-matching technique presented in the Section 2. At the trailing edge the mean-camber lines can be assimilated to a cascade of zero-stagger plates parallel to the axis. The continuity is also ensured by solving a mode-matching problem, as already reported by Roger et al. [3]. Coupling both interfaces is made possible by inserting the sound-transmission model of Section 3. 


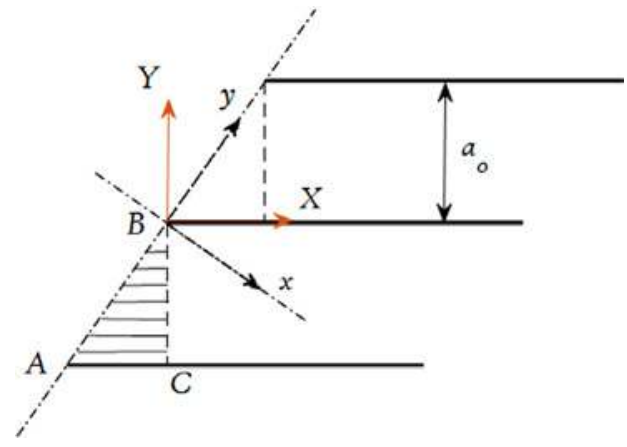

Fig. 2. References frames for matching equations at the leading-edge interface.

When downstream transmission of acoustic waves such as generated by rotor-associated noise sources is considered, the present two-dimensional approach is declined as follows.

1. The incident sound field is expanded in a series of acoustic plane-wave modes. The leading-edge response is derived for each mode using the mode-matching technique as if the vanes were semi-infinite staggered plates (Section 2). This generates upstream (reflected) modes and downstream (transmitted) modes inside the channels.

2. The downstream propagation of the transmitted modes is reproduced using Rienstra's slowly-varying duct formulation [7].

3. At the trailing-edge interface the transmitted modes are partially reflected back in the channels and transmitted downstream of the stator. This is modeled by solving another mode-matching problem taking a Kutta condition into account (Bouley et al. [5]).

4. The upstream propagation of the reflected modes in the intervane channels is described with the same slowly-varying duct approach, now accounting for possible cut-on to cut-off transitions (Ovenden [8], see details in Section 3).

5. Another staggered-plate mode-matching problem is solved to compute the secondary leading-edge scattering of the upstream traveling channel modes. Now upstream waves in the freespace and new downstream-traveling channel modes are generated.

Steps 2-5 are repeated iteratively, leading to a converged uniform solution.

The complementary problem of upstream sound transmission through the stator from hypothetical sources located downstream is also needed, for instance to model stator trailing-edge noise. In this case the procedure is initialized by deriving the response of the trailing-edge interface to upstream-propagating waves, which generates downstream reflections and upstream transmission in the channels. This is a variant of the mode-matching of step 3, from which the iterative procedure is now to repeat steps 3-4-5-2 until convergence. The present work is focussed on the separate effects of vane curvature and vane stagger angle, aimed at showing that these parameters cannot be ignored for a relevant analytical modeling of the OGV row. The effect of a constant stagger angle for flat-plate vanes is discussed in Section 2.4. The effect of camber is analyzed in Section 3 independently for the sake of a better understanding. In this case upstream propagating waves that would correspond to trailing-edge noise sources are considered in order to highlight the effect of cut-on to cut-off transitions.

\section{The staggered array of flat plates}

As a first step this section addresses the reflection-andtransmission of an incident oblique acoustic plane wave at the leading-edge interface of the stator, assuming channels of constant pitch bounded by semi-infinite staggered plates as featured in Fig. 1(b). A similar problem has been formulated for electromagnetic waves in a medium at rest by Whitehead [9], leading to a closed-form solution. The formulation is transposed to acoustic waves in the presence of a uniform flow along the plates by means of simple changes of variables. In a second step plates of finite length are considered in order to simulate a cascade of staggered vanes more consistently. It is worth noting that this part of the method could be transposed in a moving reference frame to formulate the problem of sound transmission through the blades of a rotor.

\subsection{Scattering in a medium at rest}

The acoustic scattering problem at the leading edge is solved here for a prescribed incident wave from upstream, using Whitehead's approach with rigid-wall boundary conditions. The incident, reflected and transmitted waves are described by their velocity potentials. Two reference frames are introduced, one of coordinates $(x, y)$ with the $y$ axis along the leading-edge interface, as shown in Fig. 1(b), and another one $(X, Y)$ with origin at the edge of a plate taken as reference and the $X$ axis along the plate (see Fig. 2).

Formulation of the potentials. If $\theta_{i}$ denotes the angle of incidence with respect to the $x$ direction, the potential of the incident wave is written as $\phi_{i}=A \mathrm{e}^{\mathrm{i} k\left(\sin \theta_{i} y+\cos \theta_{i} x\right)}=A \mathrm{e}^{\mathrm{i}\left(k_{y} y+k_{x} x\right)}$ with

$k_{y}=k \sin \theta_{i}=\frac{j 2 \pi}{a_{1} V}=\frac{j}{r_{0}}, \quad j \in \mathbb{Z}$.

The number of channels $V$ defines the periodicity of the system and $j$ is the index of the azimuthal order of the incident wave. $k=\omega / c_{0}$ is the acoustic wave number, $c_{0}$ is the constant sound speed and $\omega$ the angular frequency. The width of the inter-vane channels is $a_{0}=a_{1} \cos \alpha$. Therefore, the incident axial wavenumber can be noted

$k_{x}=\left[k^{2}-\left(\frac{j 2 \pi}{a_{0} V} \cos \alpha\right)^{2}\right]^{1 / 2}=k_{j}$.

The phase shift between adjacent channels only depends on the excitation and is $u=j 2 \pi / V$. The potential in the homogeneous region with reflected waves reads

$\phi_{r}=\sum_{q=-\infty}^{+\infty} R_{q} \mathrm{e}^{\mathrm{i} \alpha_{q} y} \mathrm{e}^{\mathrm{i} K_{q} x}$,

with

$\alpha_{q}=\frac{2 \pi}{a_{1} V}(j+q V), \quad K_{q}=-\sqrt{k^{2}-\alpha_{q}^{2}}$,

introducing the reflection coefficients $R_{q}$. The transmitted wave in the reference channel is better expressed in the coordinates $(X, Y)$ as

$\phi_{t}=\sum_{p=0}^{+\infty} D_{p} \cos \left(\frac{p \pi Y}{a_{0}}\right) \mathrm{e}^{\mathrm{i} K_{p} X}$,

with

$K_{p}=\sqrt{k^{2}-\left(p \pi / a_{0}\right)^{2}}$,

$D_{p}$ standing for the transmission coefficients.

Matching Equations. For a zero-stagger cascade the matching equations are simply deduced from the continuity of pressure and axial velocity as proposed by Bouley et al. [5] because explicit modal expressions are available on both sides of the interface. For a staggered cascade a different statement is chosen here. The field 
inside the so-called matching triangle $A B C$ in Fig. 2 does not need to be expressed. Green's reciprocal theorem,

$\int_{\mathcal{C}}\left(G \frac{\partial \phi}{\partial n}-\phi \frac{\partial G}{\partial n}\right) d \eta=0$,

is applied on the triangle to connect the open space and the channels, where $\mathcal{C}$ denotes the triangular contour, $\eta$ the coordinate along the contour, $\partial / \partial n$ the normal derivative and $G$ any function that satisfies the rigidity condition at the walls. The theorem can be applied with $\phi=\phi_{i}+\phi_{r}$ on the left-hand side $\mathrm{AB}$ and with $\phi=\phi_{t}$ on the right-hand side BC. Two sets of functions $G$ are selected as:

$G^{ \pm m}(X, Z)=\cos \left(\frac{m \pi Z}{a_{0}}\right) \mathrm{e}^{ \pm \mathrm{i} K_{m} X} ; \quad m \in \mathbb{N}$,

with

$K_{m}=\sqrt{k^{2}-\left(m \pi / a_{0}\right)^{2}}$.

Both fulfill the boundary conditions at the walls so that the contribution of the side AC to the integral is exactly zero. The application of Green's theorem leads to a set of linear equations on the modal coefficients of the upstream (reflected) waves and the downstream (transmitted) channel waves, written as

$\left[\underline{\mathbf{A}_{\mathbf{1}}^{+}}\right][\mathbf{R}]+\left[\mathbf{B}^{+}\right]=[\mathbf{0}]$

$\left[\underline{\mathbf{A}_{\mathbf{1}}^{-}}\right][\mathbf{R}]+\left[\mathbf{B}^{-}\right]=\left[\mathbf{D}^{\prime}\right]$

where $\mathbf{R}$ is the vector of coefficients $R_{q}$ and where

$$
\begin{aligned}
& A_{1}^{ \pm}(m, q) / A \\
& \quad=\frac{k^{2} \cos \alpha \sin \alpha \pm K_{m} \alpha_{q} \cos \alpha \mp K_{m} K_{q} \sin \alpha-K_{q} \alpha_{q}}{\left( \pm K_{m} \sin \alpha+\alpha_{q}\right)^{2}-\left(m \pi / a_{0}\right)^{2}}, \\
& B^{ \pm}(m, 1) / A \\
& =\frac{k^{2} \cos \alpha \sin \alpha \pm K_{m} \alpha_{0} \cos \alpha \mp K_{m} K_{0} \sin \alpha-K_{0} \alpha_{0}}{\left( \pm K_{m} \sin \alpha+\alpha_{0}\right)^{2}-\left(m \pi / a_{0}\right)^{2}}, \\
& D^{\prime}(m, 1)=\frac{ \pm \mathrm{i} a_{0}\left(1+\delta_{m, 0}\right) K_{q}}{(-1)^{m} \mathrm{e}^{\mathrm{i}\left( \pm a_{0} K_{m} \tan \alpha+u\right)}-1} D_{m} .
\end{aligned}
$$

The reflection coefficients are obtained by solving Eq. (3) by matrix inversion. The transmission coefficients are directly deduced from Eq. (4). The same technique can be applied to calculate the scattering of a set of prescribed phase-shifted modes propagating upstream in the channels and being partially transmitted in the open space; in this case no incident wave from upstream is involved. Furthermore, at the price of a simple change of variables, a similar formulation can be written in the complementary case of the trailing-edge interface of staggered plates extending to infinity upstream. This has been implemented to produce the results of Section 2.4.

\subsection{Scattering in a uniform mean flow}

In the presence of a uniform mean flow, the Prandtl-Glauert transform is used to derive an equivalent sound diffraction problem in a stationary fluid, with modified parameters. The mean flow is assumed of Mach number $M_{0}$, so that the wave potentials have to be solutions of the convected Helmholtz equation:

$$
\frac{\partial^{2} \phi}{\partial Y^{2}}+\left(1-M_{0}^{2}\right) \frac{\partial^{2} \phi}{\partial X^{2}}+2 \mathrm{i} k M_{0} \frac{\partial \phi}{\partial X}+k^{2} \phi=0
$$

in the coordinate system $(X, Y)$ of Fig. 2 with $\beta^{2}=1-M_{0}^{2}$ and with the boundary conditions

$$
\frac{\partial \phi}{\partial Y}=0, \quad X \in\left[\mu a_{0} \tan \alpha,+\infty\left[, \quad Y=\mu a_{0}, \quad \mu \in \mathbb{Z} .\right.\right.
$$

The transform is written as $\tilde{X}=X / \beta, \tilde{k}=k / \beta$ and $\Phi=\mathrm{e}^{\mathrm{i} \tilde{k} M_{0} \tilde{X}} \phi$ and leads to the ordinary Helmholtz equation

$\frac{\partial^{2} \Phi}{\partial Y^{2}}+\frac{\partial^{2} \Phi}{\partial \tilde{X}^{2}}+\tilde{k}^{2} \Phi=0$

and the boundary conditions:

$\frac{\partial \phi}{\partial Y}=0, \quad \tilde{X} \in\left[\mu a_{0} \tan \tilde{\alpha},+\infty\left[, \quad Y=\mu a_{0}, \quad \mu \in \mathbb{Z}\right.\right.$

introducing $\tan \tilde{\alpha}=\tan \alpha / \beta$. In the modified variables, the incident wave reads:

$\phi_{i}=A \mathrm{e}^{\mathrm{i} \tilde{k}\left(\cos \tilde{\theta}_{i} \tilde{x}+\sin \tilde{\theta}_{i} \tilde{y}\right)}$.

If the incident wave is of angle $\theta_{i}$ with respect to $x$ in the original diffraction problem with flow, the modified angle of incidence $\tilde{\theta}_{i}$ is defined as:

$\sin \tilde{\theta}_{i}=\frac{\beta \sin \theta_{i}}{1+M_{0} \cos \theta_{i}} ; \quad \cos \tilde{\theta}_{i}=\frac{M_{0}+\cos \theta_{i}}{1+M_{0} \cos \theta_{i}}$.

As a result, the Prandtl-Glauert transform allows solving an equivalent sound diffraction problem without flow for a cascade of vanes with a larger stagger angle $\tilde{\alpha}>\alpha$ and a larger wave number $\tilde{k}>k$. The same procedure as described in the previous subsection can therefore be applied. Next performing the inverse transform, the diffracted potential fields are obtained.

\subsection{Extension to a staggered array of finite-length flat plates}

The same methodology can be applied to a cascade of staggered plates of finite chord length by adding the effect of the trailingedge interface. For this it is assumed that adjacent vanes overlap significantly so that both interfaces can be treated separately in an iterative procedure. Another non-overlapping triangle is defined at the trailing edges, symmetric of the triangle $A B C$ in Fig. 2. The downstream-traveling channel waves generated by the initial scattering are scattered at the trailing-edge interface, producing reflected waves upstream in the channels and transmitted oblique waves downstream of the stator in open field. The back-and-forth propagation of waves in the channels is reproduced this way, the convergence being obtained after a couple of iterations. This flatplate assumption ignores the effect of vane curvature but the effect of stagger angle with finite chord can be investigated quite simply. Moreover the flow must be assumed uniform in the whole domain, which would be acceptable in a relative reference frame for a rotor of very small camber but is not compatible with the swirl recovery of OGV. Typical results are discussed in the next section.

\subsection{Sample results}

As a test case, the mode-matching technique based on Green's reciprocal theorem and the Prandtl-Glauert transform is applied in this section for two periodic cascades of flat plates, one with a zero stagger and the other one with a stagger angle of $20^{\circ}$. Both are impinged by the same oblique acoustic wave. The parameters of the simulation, selected to expectedly generate the axial planewave mode of order zero, are given below:

- Number of vanes: $V=10$

- Vane chord: $c=4.5 \mathrm{~cm}$

- Vane-to-vane spacing: $a_{1}=2 \mathrm{~cm}$

- Radius of the unwrapped cascade: $r_{0}=80 \mathrm{~mm}$

- Mach number: $M_{0}=0.2$

- Dimensionless incident wavenumber (Helmholtz number): $k c=6.8$ (at $19 \mathrm{kHz}$ )

- Equivalent azimuthal mode order of the incident wave (number of lobes): $j=V=10$. 

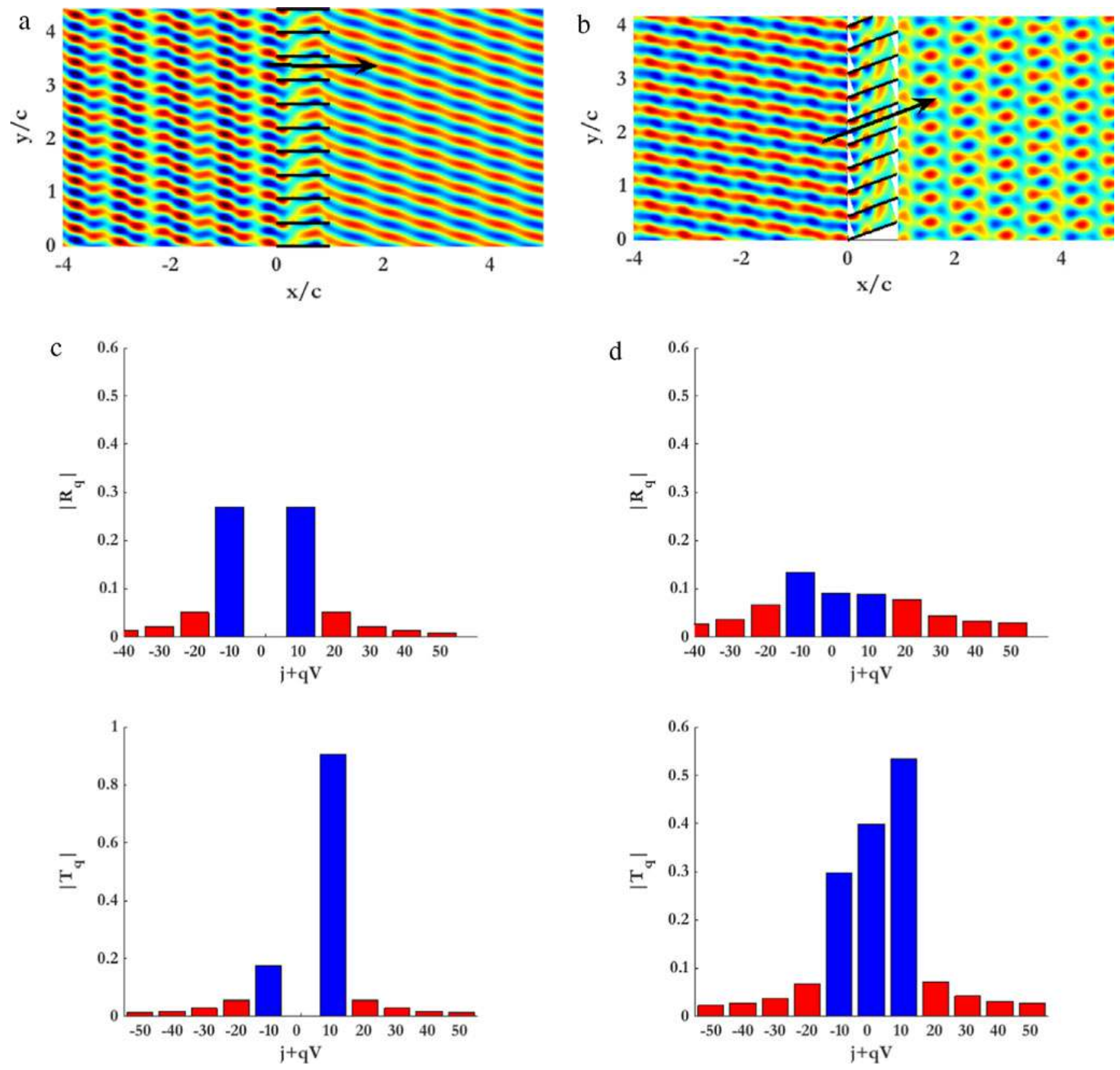

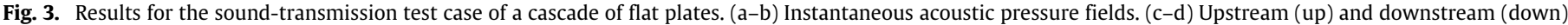
modal coefficients for the unstaggered (c) and staggered (d) configurations $(j=10, V=10)$. Effective orders $j+q V$ as abscissa in diagrams (c) and (d).

The results are shown in Fig. 3. The instantaneous acoustic pressure patterns of the combined incident and scattered waves are presented in Fig. 3(a) and (b). The amplitudes of the reflected and transmitted modes propagating upstream and downstream of the stator, $\left|R_{q}\right|$ and $\left|T_{q}\right|$ respectively, are displayed as bar-graphs in Fig. 3(c) and (d). The white triangles in Fig. 3(b) correspond to the matching triangles. They reduce to infinitesimal areas for the zero-stagger configuration in Fig. 3(a). The acoustic pressure in the triangles is not needed when calculating the scattered field of interest, similarly to previous cascade-response studies from Glegg [10]. Both configurations exhibit the same qualitative pattern of reflected and transmitted waves: the scattered cut-on mode orders $j+q V$ are $-10,0$ and $10(q=-2,-1,0)$. All other modes are cut-off, decaying exponentially. However the magnitudes of the cut-on modes significantly differ, as shown by the bar-graphs in Fig. 3(c) and (d). The reflected acoustic field for the zero-stagger case is a symmetric pattern with respect to the tangential direction: the magnitudes of the scattered orders $j+$ $q V= \pm 10(q=-2$ or 0$)$ are equal and that of the axial plane-wave mode $(q=-1)$ is zero. Because the equivalent acoustic sources of the cascade of vanes are dipoles oriented in the normal direction of the plates, phase-shifted by $j 2 \pi / V$, the axial plane-wave mode cannot be excited. In contrast Fig. 3(d) confirms that the staggered cascade is able to produce this mode because the equivalent dipoles are now inclined: they have a non-zero axial projection. The reflected acoustic pattern is no longer symmetric and the major part of the reflected energy propagates in the tangential direction opposite to the incident wave, which seems to be quite consistent with the stagger angle. Concerning the transmitted waves, the dominant mode of azimuthal order -10 propagates in the opposite tangential direction with respect to the incident wave for the zero-stagger case, whereas the cut-on mode of order $j+q V=$ 10 propagates in the same direction. For the staggered case, the modes combine differently in such a way that the two modes 


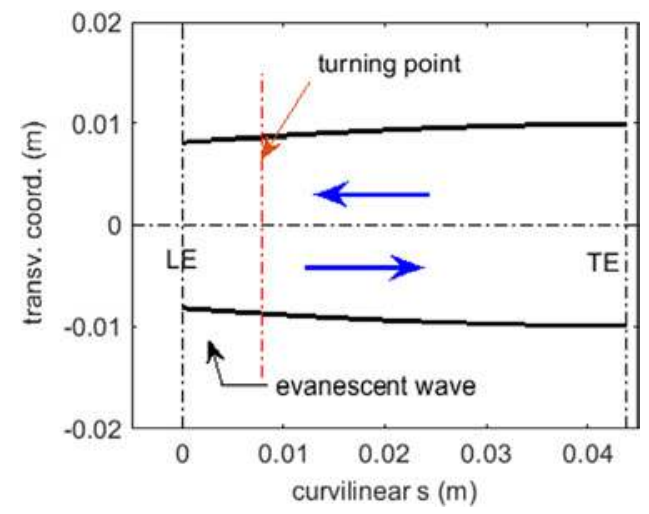

Fig. 4. Illustration of the unbent inter-vane channel and of the turning point of a channel mode. Primary and reflected waves featured by arrows.

$j+q V= \pm 10$ have nearly equal amplitudes, on the one hand, and that the axial plane-wave mode has a noticeable contribution, on the other hand. Finally it has been verified that the channel modes of orders $m=0,1$ and 2 are cut-on in both configurations and that all higher-order modes are cut-off.

The present analysis confirms that the stagger angle of a cascade of flat plates has a significant impact on the scattered pressure field. However both configurations displayed in Fig. 3(a) and (b) could be considered as acceptable simplifications of the true stator geometry. More precisely each is more realistic at only one interface. This suggests that the most relevant strategy is to treat the scattering problem at the leading-edge with an array of semi-infinite staggered flat plates and at the trailingedge with an array of semi-infinite zero-staggered flat plates. The only way of making both compatible is to introduce the curvature of the inter-vane channels in the sound propagation model, for the sake of completeness and consistency. In fact, ignoring the blade curvature would be acceptable in the tip region of a fan or compressor rotor for many axial-flow designs. But outlet guide vanes are always significantly curved because they have to ensure the mean-flow deviation that corresponds to the swirl recovery. The proposed technique to introduce the curvature in the formulation is presented in Section 3 as a separate topic.

\section{Transmission through inter-vane channels}

The results of the previous section stress that the inclination of the vanes is a crucial parameter in the mode-matching technique based on the assumption of equivalent flat plates. The next step is to include the true curvature of the vanes as shown in Fig. 1(b) into account. This is achieved at the price of an approximation with a multiple-scale analysis, considering the overlapping part of the inter-vane channel (excluding the matching triangle $A B C$ in Fig. 1(b)) as an equivalent straight duct of slowly varying cross-section, the actual curvilinear coordinate $s$ being assimilated to an axial coordinate as illustrated in Fig. 4. In this section sound transmission is considered for an upstream propagating wave that originates from the stator trailing-edge interface ('TE' in the figure). This choice is made to highlight some effects of the channel expansion, such as the possible total reflection of an upstream-traveling wave at some turning point, involved in the upstream transmission of cascade trailing-edge noise. The same analysis could be developed for incident waves in the opposite direction.

For a channel height $a_{1}$ at the outlet (trailing edge), the channel height between points $B$ and $C$ in Fig. 1(b) at the inlet (section ' $L E$ ' in Fig. 4) normal to the vanes is $a_{0}^{\prime}$ such that:

$\frac{a_{0}^{\prime}}{R_{c}}=\left(1+\frac{a_{1}}{R_{c}} \cos \alpha\right)-\sqrt{1-\left(\frac{a_{1}}{R_{c}} \sin \alpha\right)^{2}}=a_{0}+e$ for a curvature radius $R_{c}$ of the vane arc, $e$ being the small error mentioned in Section 1, and the effective length of an unwrapped inter-vane channel is approximated as

$L \simeq\left(R_{c}-\frac{a_{0}}{2}\right) \alpha$.

Over this length the channel height varies continuously. The approximation neglects bent-duct effects on the propagation of acoustic modes but accounts for the diverging flow in the channel induced by the curvature of the vanes. It is accepted as the simplest way of accounting for a realistic stator geometry, based on the idea that the variable channel height is the dominant feature. The longitudinal variation of cross-section is then written as

$a(s)=\frac{a_{0}}{\cos \alpha} \cos \left(\frac{s}{R_{c}}-\alpha\right)$

if the origin of $s$ is taken at the section $\mathrm{BC}$.

In this model the chord length $c$ of the vanes is expressed as $c=\sqrt{2} R_{c} \sqrt{1-\cos \alpha}$, which means that the radius of curvature can be of the same order of magnitude as the radius $r_{0}$ of the unwrapped cut for typical values of $V$ and of the ratio $c / a_{1}$.

Rienstra's multiple-scale analysis [7] can now be applied. Only the key steps are summarized in this work for conciseness. The transverse modes are the same cosine modes as for the twodimensional channel of constant height except that now the amplitude varies according to the factors

$N(s)=Q / \sqrt{k \sigma(s)}$,

$\sigma(s)=\sqrt{1-\beta^{2}(s)\left(\frac{m \pi}{k a_{0}}\right)^{2}\left(\frac{a_{0}}{a(s)}\right)^{2}}$.

Their shape varies according to the local channel height noted $a(s)$. $Q$ is an amplitude parameter related to the factor $A$ in the previous section. The relationship between $a(s)$ and other quantities allows determining the wavenumber parameter as

$\mu(s)=-\frac{k M(s)}{\beta^{2}(s)} \pm \frac{1}{\beta^{2}(s)}\left(k^{2}-\left[\frac{m \pi \beta(s)}{a(s)}\right]^{2}\right)^{\frac{1}{2}}=\mu_{c} \pm \mu_{p}$

with $\beta(s)=\sqrt{1-M^{2}(s)}, \mu_{c}$ and $\mu_{p}$ standing for the convective and propagating parts, respectively. If the mean flow is assumed incompressible the local Mach number is simply

$M(s)=\frac{U_{a}}{c_{0} \cos \alpha} \frac{a_{0}}{a(s)}$.

A more general statement could be proposed for a compressible mean flow by introducing variable density and speed of sound depending on the coordinate $s$ [7]. In a turbomachinery stage this requires that the mean-flow is previously determined. This refinement is discarded from the present work dedicated to the feasibility of the approach.

Neglecting the curvature of the inter-vane channels is a questionable simplification that has been justified by an approximate analysis. The latter is not detailed for conciseness but its principle is briefly outlined in this paragraph. A portion of a bent duct defined by concentric walls of limited arcs has been considered to mimic an inter-vane channel ignoring its height variation. Sound propagation in the bent duct was described by the Helmholtz equation in polar coordinates. Assuming that the radial distance from the mean centerline of the bent duct remains small when compared to its mean curvature radius, the ordinary Helmholtz equation was recovered by a factorization and a change of variables, assimilating the radial and tangential coordinates to Cartesian coordinates. The standard solving led to an analytical expression for the transverse bent-duct modes. Expanding these modes on the set of usual cosine 
modes showed that only one mode strongly dominates whereas all others are generated at a negligible level, at least for a set of parameters representative of the technology of Fig. 1. Furthermore the curvature was shown to cause a slight shift of the cut-off frequencies of the modes, also found negligible. This confirms that the cosine modes can still be used to describe the sound field in the bent duct, simply projecting from the unwrapped Cartesian coordinates onto the polar coordinates. The same simplification has been accepted for a slowly varying channel height in the presence of flow. It is worth noting that the curvature could be combined with the varying channel height using Brambley \& Peake's analysis [11] or the multimodal expansion proposed by Félix \& Pagneux [12]. The straight-duct approximation is preferred for its simplicity.

If an upstream-propagating mode experiences transition at some location $s_{t}$ it is reflected back and the transmission farther to the right takes the form of an evanescent wave. The corresponding field before the turning point $\left(s>s_{t}\right)$ reads

$$
\begin{aligned}
\phi= & N(s) \cos \left(\frac{m \pi y}{a(s)}\right) A_{0} \mathrm{e}^{-\mathrm{i} \int_{s_{t}}^{s} \mu_{c} \mathrm{~d} \xi} \\
& \times\left\{\mathrm{e}^{-\mathrm{i} \int_{s_{t}}^{s} \mu_{p} \mathrm{~d} \xi}+\mathrm{i} \mathrm{e}^{\mathrm{i} \int_{s_{t}}^{s} \mu_{p} \mathrm{~d} \xi}\right\}
\end{aligned}
$$

and the transmitted field beyond the transition $\left(s<s_{t}\right)$ is expressed as

$\phi=N(s) \cos \left(\frac{m \pi y}{a(s)}\right) A_{0} \mathrm{e}^{-\mathrm{i} \int_{s_{t}}^{s} \mu_{c} \mathrm{~d} \xi} \mathrm{e}^{-\int_{s_{t}}^{s} \mu_{p} \mathrm{~d} \xi}$,

$A_{0}=\mathrm{e}^{-\mathrm{i} \int_{s_{0}}^{s_{t}} \mu(\xi) \mathrm{d} \xi}$,

the abscissa $s_{0}$ being taken at the channel trailing-edge interface. This means that the reflection and transmission coefficients associated to the transition are $i$ and 1 , respectively. The solution is singular at the transition because of the definition of $N(s)$ in which the denominator goes to zero. If the transition takes place close to one end of the channel the singularity can give the mode an abusively high amplitude. This is why the singularity needs to be removed by implementing the uniformly valid solution proposed by Ovenden [8] for a cut-on to cut-off transition, as

$$
\begin{aligned}
\phi= & \frac{2 \sqrt{\pi} Q e^{\mathrm{i} \pi / 4}}{\sqrt{k a_{1}}} \cos \left(\frac{m \pi y}{a(s)}\right) \\
& \times\left[\frac{-3}{2 \sigma^{3}(s)} \int_{s_{t}}^{s} \frac{\left(k a_{1}\right) \sigma(\xi)}{\beta^{2}(\xi)} \mathrm{d} \xi\right]^{1 / 6} \\
& \times A_{i}\left\{\left[\frac{3 \mathrm{i}}{2} \int_{s_{t}}^{s} \frac{\left(k a_{1}\right) \sigma(\xi)}{\beta^{2}(\xi)} \mathrm{d} \xi\right]^{2 / 3}\right\} \mathrm{e}^{-\mathrm{i} \int_{s_{t}}^{s} \mu_{c} \mathrm{~d} \xi}
\end{aligned}
$$

in which $A_{i}$ is the Airy function. A slightly different expression is found in the reference paper, valid for a compressible mean flow. Note that cut-off to cut-on transitions are not considered in the present work. They would correspond to sound-energy pumping by an equivalent acoustic tunnel effect from the upstream open field. No consistent formulation is available for this effect but no evidence of it has been reported. It is therefore assumed that the excitation of a cut-off mode at the stator leading-edge does not give rise to effective propagation farther downstream.

Sample results (in arbitrary amplitude units) in cases for which cut-on to cut-off transition occurs are presented in Figs. 5 and 6 for two sets of parameters. The first one reproduces the test in Fig. 3; it corresponds to a low-speed ducted fan at very high frequency $(19 \mathrm{kHz})$. The second one is more representative of the outlet guide vanes of a turbofan engine at reduced power. The upper plots (a) show instantaneous pressure maps and the lower plots (b) display the associated pressure-amplitude profiles along the wall, showing maxima and nodes of the standing wave pattern produced
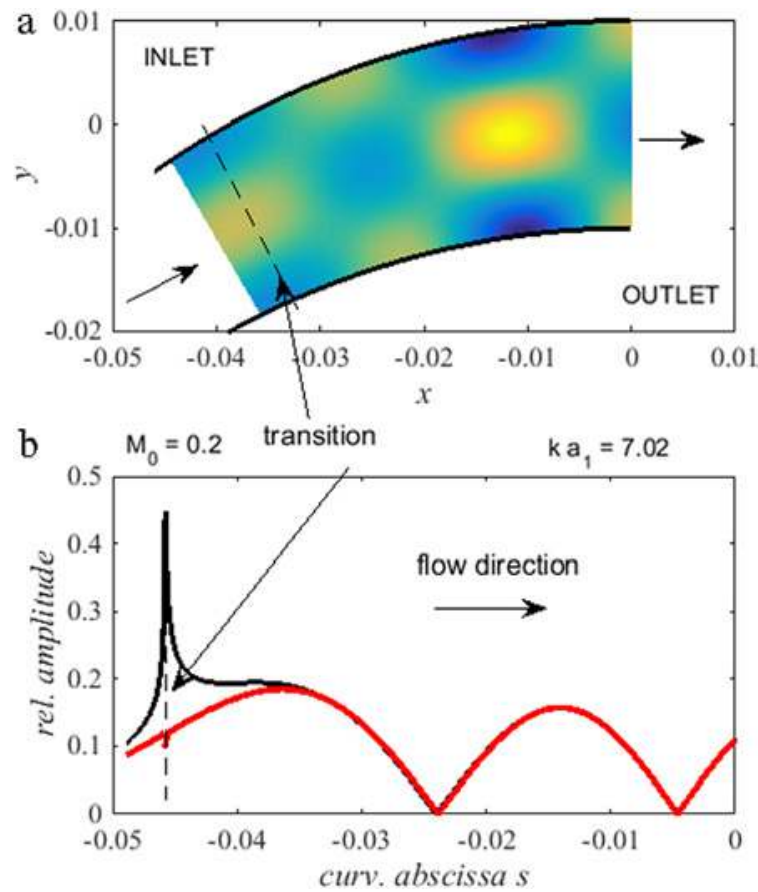

Fig. 5. Transition of the upstream channel mode $m=2$ for $k a_{1}=7.02, M_{0}=0.2$ (a) Instantaneous pressure map. (b) Amplitude profile along the wall. Initial (black) showing the peaks at the transition abscissa; regularized (red). (For interpretation of the references to color in this figure legend, the reader is referred to the web version of this article.)
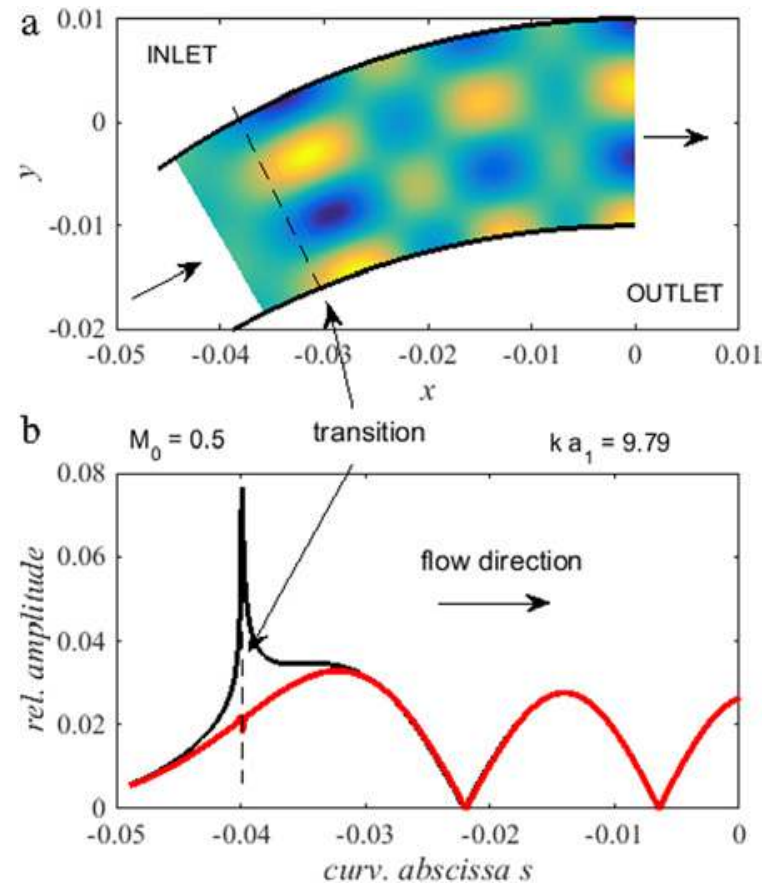

Fig. 6. Transition of the upstream channel mode $m=3$ for $k a_{1}=9.79, M_{0}=0.5$ (a) Instantaneous pressure map. (b) Amplitude profile along the wall. Initial (black) showing the peaks at the transition abscissa; regularized (red). (For interpretation of the references to color in this figure legend, the reader is referred to the web version of this article.)

by the combined primary and reflected waves. The black lines of the plots (b) stand for the initial, singular solutions that exhibit a strong divergence around the abscissa of the transition (combined Eqs. (5) and (6)). In contrast the uniformly valid Ovenden's solution (Eq. (7)) plotted in red produces smoothed profiles. The transition 
is indicated by the dashed lines. In the first case (Fig. 5) dealing with the mode $m=2$ the transition takes place quite close to the inlet of the channel and the exponential decay is only moderate. This means that the mode contributes significantly to the matching at the leading edge, even though it is cut-off. Moreover the initial and regularized solutions differ at the inlet, which means that ignoring the regularization would lead to a bad estimate of the mode amplitude. It must be noted that this mode was cut-on all along the channels in the test case of Fig. 3 assuming equivalent flat plates, which is an abusive simplification. In the second case (Fig. 5) the evanescent wave upstream of the transition has significantly decayed at the inlet cross-section where the initial and regularized solutions coincide. The mode contributes weakly to the matching at the leading edge; but its reflection is anyway determinant for the matching at the trailing-edge interface. Obviously the possible transition of the primary wave must be included for a correct statement of the matching problem. At the outlet this is typically achieved by always considering the upstream and downstream waves together with equal amplitude and the proper phase shift, when a transition is predicted for a mode.

\section{Conclusions}

Two aspects of a new methodology for the analytical prediction of sound transmission through axial-flow outlet guide vanes have been presented in this work, aimed at reproducing the effects of both vane stagger and vane camber. Adjacent vanes must significantly overlap in order that a true waveguide behavior takes place in the overlapping part of the inter-vane channels. A standard mode-matching technique is applied at the trailing-edge interface where the vanes are assumed semi-infinite plates of zero stagger. Another mode-matching formulation is applied at the leadingedge interface to cope with the area where adjacent vanes do not overlap; here the vanes are assumed staggered semi-infinite plates. Both matching problems are solved iteratively to reproduce the back-and-forth wave motions associated with the finite chord length. Between both interfaces the curved inter-vane channels are assimilated to straight ducts of slowly varying width and the effect of that variation on sound propagation is taken into account by means of a multiple-scale analysis. The latter acts as a transfer function between the leading-edge and trailing-edge interfaces. It is shown by separate inspection that both the vane inclination and the vane camber have a significant effect on sound transmission. Though only detailed for acoustic wave transmission in a two-dimensional context the approach is believed promising because of its large possibilities of extension. For instance the mode matching can be formulated in a three-dimensional annular geometry. Furthermore incident hydrodynamic excitations can be considered instead of acoustic waves to also formulate the problem of sound generation by wake impingement on a stator. These extensions are presently under development. It is worth noting that away from the cascade upstream and downstream and locally in the vicinity of each interface, the mean-flow conditions must be uniform; but the slowly-varying duct approach tolerates meanflow conditions that vary continuously along the curved channel axis even though they have to be constant over a cross-section normal to that axis. This typically allows taking into account compressibility effects and not only the deviation from swirling flow to axial flow.

\section{Acknowledgments}

The present developments have been partially made in the EC-funded project ACP2-GA-2012-314066 IDEALVENT (Integrated Design of Optimal Ventilation Systems for Low Cabin and Ramp Noise). The authors are grateful to Michael Bauerheim for his contribution in the multiple-scale analysis.

\section{References}

[1] M.E. Goldstein, Aeroacoustics, McGraw-Hill, 1976

[2] J.E. Ffowcs Williams, D.L. Hawkings, Sound generation by turbulence and surfaces in arbitrary motion, Philos. Trans. R. Soc. Lond. Ser. A Math. Phys. Eng. Sci. 264 (1151) (1969) 321-342.

[3] M. Roger, B. François, S. Moreau, Cascade trailing-edge noise modeling using a mode-matching technique and the edge-dipole theory, J. Sound Vib. 382 (2016) 310-327.

[4] H. Posson, S. Moreau, M. Roger, Broadband noise prediction of fan outlet guide vane using a cascade response function, J. Sound Vib. 330 (25) (2011) 6153-6183.

[5] S. Bouley, B. François, M. Roger, H. Posson, S. Moreau, On a mode-matching technique for sound generation and transmission in a linear cascade of outlet guide vanes, in: 21st AIAA/CEAS Aeroacoustics Conference, Vol. 2825, 2015.

[6] J. de Laborderie, V. Blandeau, T. Node-Langlois, S. Moreau, Extension of a fan tonal noise cascade model for camber effects, AIAA J. 53 (4) (2015).

[7] S.W. Rienstra, Sound transmission in slowly varying circular and annular lined ducts with flow, J. Fluid Mech. 380 (1999) 279-296.

[8] N.C. Ovenden, A uniformly valid multiple scales solution for cut-on cut-off transition of sound in flow ducts, J. Sound Vib. 286 (1) (2005) 403-416.

[9] E.A.N. Whitehead, The theory of parallel-plate media for microwave lenses, Proc. IEE-Part III: Radio Commun. Eng. 98 (52) (1951) 133-140.

[10] S.A.L. Glegg, The response of a swept blade row to a three-dimensional gust, J. Sound Vib. 227 (1) (1999) 29-64.

[11] E.J. Brambley, N. Peake, Sound transmission in strongly-curved slowlyvarying cylindrical and annular lined ducts with flow, in: 12th AIAA/CEAS Aeroacoustics Conference, Vol. 2582, 2006

[12] S. Félix, V. Pagneux, Sound propagation in rigid bends: a multimodal approach, J. Acoust. Soc. Am. 110 (3-1) (2001) 1329-1337. 